\title{
Le Management Stratégique De La Recherche Dans Les Universités Publiques Au Sénégal : Cas De l'Université Cheikh Anta Diop De Dakar
}

\author{
Pr. Mouhamed El. Bachir Wade, \\ Dr. Ousmane Tanor Dieng, \\ Dr. Massamba Samb,
}

Enseignant-chercheur, Université Cheikh Anta Diop de Dakar-Sénégal, Faculté des Sciences Economiques et de Gestion

Doi:10.19044/esj.2019.v15n31p184 URL:http://dx.doi.org/10.19044/esj.2019.v15n31p184

\section{Resume}

Les universités font face à une évolution rapide de leur environnement. Les autorités étatiques (à qui l'université publique fait supporter de gros investissements) s'interrogent sur les voies pour rendre ces espaces de production de savoirs et d'innovations plus compétitifs. Les auteurs sensibles à cette préoccupation n'hésitent pas à mettre le focus, dans leur analyse, sur l'activité de recherche à l'université notamment la manière dont celle-ci est managée. Cette contribution prend prétexte de cette préoccupation en examinant les dysfonctionnements dans le management stratégique de la recherche universitaire au Sénégal. L'étude prend comme cas empirique l'université Cheikh Anta Diop de Dakar, la plus importante université au Sénégal. Les données recueillies dans cette institution ont fait l'objet d'une analyse de contenu à l'aide du logiciel Tropes. Les résultats révèlent que le management de la recherche dans cette institution fait face à des défis majeurs dont celui relatif à la nécessité de construire une vision prospective qui arrime durablement le système de recherche universitaire actuel aux politiques nationales de développement.

Mots clés : Recherche, Management, Management stratégique de la recherche, Dysfonctionnements 


\title{
The Strategic Management of Research in Public Universities in Senegal: Case of the University Cheikh Anta Diop of Dakar
}

\author{
Pr. Mouhamed El. Bachir Wade, \\ Dr. Ousmane Tanor Dieng, \\ Dr. Massamba Samb, \\ Enseignant-chercheur, Université Cheikh Anta Diop de Dakar-Sénégal, \\ Faculté des Sciences Economiques et de Gestion
}

\begin{abstract}
Universities are facing a fast change of their environment. The public authorities (to which the public university make them bear large-scale investments) are questioning ways and means to make these institutions more competitive. Authors do not hesitate to focus, in their analysis, on the research activity at the university mainly on how the latter is managed. We tried in this paper to analyze the dysfunctions of the strategic management of university research in Senegal. The university Cheikh Anta Diop of Dakar, the most important university in Senegal, is chosen as empirical case. The data collected in this institution were exploited through a content analysis using the Tropes software. The results reveal that the management of research in this institution faces major challenges, including the need to build a forwardlooking vision that permanently links the current university research system to national development policies.
\end{abstract}

Keywords: Research, Management, Strategic management of research, Dysfunctions

\section{Introduction}

Les structures de recherche composent avec nombre de défis qui ont pour appellation : «devenir de la recherche », «fuite des cerveaux », « crise de la recherche » etc. (Theys, 2007). Si ces défis se posent avec acuité dans le contexte de l'université, c'est parce qu'elle est confrontée à l'évolution rapide de son environnement résultant des contraintes de financement, des impacts de la mondialisation, de la compétitivité interuniversitaire etc. (Connell, 2004). Par la force des facteurs précités, les rapports entre pouvoirs publics et institutions universitaires vont connaître un véritable tournant. Celles-ci sont 
soumises de plus en plus à des contraintes «de transparence, de responsabilisation et d'imputabilité » (Atallah et Boyer, 2002, p.1). Autour de ces notions évoquées par ces auteurs se sont développés des travaux orientés les uns vers la gestion de la recherche (GER) et les autres vers la gouvernance de la recherche (GVR).

Les travaux sur la GER réalisés pour la plupart dans les pays développés ont tenté d'examiner les caractéristiques de la GER particulières à certains établissements de la zone en vue, entre autres, de partager des expériences sur certaines pratiques (Taylor, 2006).

En Afrique de l'Ouest et du Centre (AOC), les travaux ont plutôt porté sur le thème de la gouvernance de la recherche (GVR) dans les universités de la région. L'objectif était de documenter les caractéristiques de la GVR et d'identifier les faiblesses et les pratiques jugées comme étant des plus efficaces.

S'il est un sujet sur lequel les travaux sont des plus rares, un sujet qui, comme la GVR et la GER, s'intéresse à l'activité scientifique des établissements d'enseignement supérieur et de recherche, c'est bien le management de la recherche. A tel point que Theys (ibid.) en est arrivé à parler de la nécessité de combler un vide. Ce papier tente de répondre à cet appel en posant un regard critique sur la manière dont la recherche est managée au Sénégal. La question qui oriente la réflexion est posée ainsi : quels sont les dysfonctionnements observables dans le management stratégique de la recherche à l'UCAD? L'orientation de la question n'a rien « d'alarmiste » lorsqu'on se positionne dans l'école de Savall et Zardet (2010, p.255). Selon ces auteurs, l'analyse dysfonctionnelle «possède un fort pouvoir incitatif de stimulation des comportements humains, que ne possède pas un diagnostic classique du type Points forts/Points faibles, où la satisfaction sur les points forts efface le relief des points faibles ». Qui plus est, les dysfonctionnements sont observables dans toutes les organisations. Seulement, ils sont plus prononcés dans certaines organisations que dans d'autres (Bonnet, 2003).

Le plan adopté pour traiter la question sus déclinée démarre par un cadrage sur le bienfondé de s'arrêter sur le management de la recherche dans le contexte de l'UCAD. L'attention est ensuite centrée sur le concept de management stratégique de la recherche qui est une notion centrale dans cette étude. Le sens du concept de dysfonctionnement pris en compte dans la question ci-dessus est aussi explicité. La démarche méthodologique est ensuite exposée avant de présenter et de discuter les résultats.

\section{L'Université Cheikh Anta Diop : un «terrain » pertinent pour la problématique du sujet.}

L’analyse des plans stratégiques des universités publiques du Sénégal donne une certaine idée sur les intentions que ces institutions prêtent à la 
recherche. L'on note à travers ces plans que ces universités aspirent, par le biais de la recherche, à contribuer au développement (local, national) et faire avancer les connaissances. Des raisons objectives méritent qu'on s'arrête sur la situation de l'UCAD. L'institution représente près de $60 \%$ de la production scientifique du pays. Elle accueille dans ses (79) formations doctorales et (169) unités de recherche ${ }^{26}$ les doctorants des autres universités publiques sénégalaises. L'obligation de promouvoir la recherche fondamentale et la recherche appliquée est établie sous ces termes par les textes de l'institution : «L'Université de Dakar est également chargée de promouvoir la recherche scientifique fondamentale, et de coopérer à la recherche appliquée, pour la République du Sénégal comme pour les Etats africains qui le désirent. Elle remplit ces missions en étroite coopération avec les autres universités africaines, ainsi qu'avec les Etats étrangers liés au Sénégal par un accord de coopération. ». Alors que ces considérations dénotent l'ampleur des attentes de l'Etat du Sénégal à l'endroit de l'UCAD, autorités universitaires et partenaires au développement admettent que la recherche universitaire " manque d'efficacité dans son organisation et son administration » (Camara et Touré, 2010, p.2). Sans forcer le trait, comment « disculper » le management là où prend place une question d'organisation? Une interrogation très légitime lorsqu'on sait que le management est à l'organisation «ce que la médecine est à l'anatomie » (Dayan et al. 2004, p.82). Le plan stratégique de l'UCAD peut être convoqué pour appuyer le bien-fondé de cette question. L'on apprend du plan que l'UCAD est confrontée à des contraintes et pesanteurs qui hypothèquent sa compétitivité en matière de recherche. Le classement 2016 effectué par le Webometrics Ranking of Word Universities corrobore cette perte de vitesse. Ledit classement positionne l'UCAD (la plus grande université du Sénégal) assez loin (31ème place) dans le top 50 des universités africaines les plus dynamiques sur le plan de la recherche. Il est à rappeler que le Webometrics Ranking of Word Universities évalue chaque établissement en considérant, entre autres, sa contribution à la connaissance moderne et le nombre de ses travaux de recherche. Même pour les universités non convaincues des critères de classement de Webometrics, elles gagneraient, dans un contexte de contraintes financières doublé d'une demande de formation en expansion à laquelle elles sont confrontées, à remettre toujours en cause leurs pratiques managériales et à s'inspirer des exemples de réussites. D'autant plus que comme le soulignent Robbins et Decenzo (2006), dans un contexte de concurrence, les organisations qui tirent leurs épingles du jeu devant celles en mal de compétitivité le doivent à un meilleur management.

\footnotetext{
${ }^{26}$ Source : Direction de la recherche de l'UCAD
} 


\section{Le concept de management stratégique de la recherche (MSR désormais) : vers une définition.}

Selon Butaré et Zoundi (2005, p.14), la recherche « réfère à l'activité de génération ou d'acquisition de nouvelles connaissances ou de nouvelles utilisations des connaissances en vue de l'intérêt général ou privé pour accrô̂tre la production nationale ou pour améliorer les conditions de vie des groupes ou des individus. ». Dans cette acception, on distingue en filigrane la recherche fondamentale (dont la visée est perceptible au travers de l'idée mise en italique) et la recherche appliquée (dont la finalité est mise en relief dans l'idée mise en gras) soit deux types que l'université peut combiner quand d'autres institutions en sont incapables (Altbach, 2007). Quel que soit le type de recherche, l'exercice commande rigueur et efforts de coordination (Wacheux, 1996). Cette idée a une valeur péremptoire chez Usinier et al. (2000, p.97) qui précisent que « la recherche ne constitue pas une série d'actes de pensée, strictement isolés les uns des autres. Il faut penser les liens de cohérence entre les différentes étapes du processus de recherche,... ». La dernière idée dans cette affirmation insiste en filigrane sur l'importance de la coordination (gage de cohérence) entre les étapes du processus de recherche.

Insister sur la coordination dans le déroulement de la recherche c'est reconnaître qu'elle implique plusieurs opérations. Ce qui a amené Savall et Zardet (2004) à assimiler l'activité de recherche au pilotage d'un projet stratégique où «le chercheur se doit de définir ses objectifs à moyen et long termes, d'analyser son environnement, et de choisir ensuite son cheminement, des moyens, des actions et des indicateurs de pilotage pour atteindre ses objectifs » (ibid., p.163). Le rapprochement est permis entre la métaphore du pilotage (à laquelle nous adhérons) dont font allusion ces auteurs et la conception de Plane (1994) sur le management. Ce dernier assimile le management à une manière de piloter encadrée par trois principes : le principe d'énergie (fixer des objectifs, prendre des décisions et réaliser des actes de mise en œuvre), le principe de coopération (faire partager le contenu des décisions) et le principe d'instrumentation (disposer d'un tableau de bord de pilotage). Ce qui ressort comme très important de ces enseignements de Plane (1994) c'est le degré de compatibilité entre sa conception du management (qu'il décrit comme une manière de piloter) et l'acception décrivant la recherche comme un pilotage stratégique.

De Latt et Barré (2007) font partie des rares auteurs à tenter d'appréhender le management de la recherche. Pour ces auteurs, le management de la recherche va de la définition des stratégies jusqu'à l'évaluation des résultats et il cherche à trouver les bons compromis entre :

-« la créativité et la résolution des problèmes »: en d'autres mots, qu'est ce qui doit guider la recherche ? La réflexion sur le choix des objectifs associés à la recherche qui est conduite s'invite dans cette question. 
- «la fermeture et l'ouverture sur la société »: on peut convoquer ici les réflexions de Gibbons et al. (1994) qui distinguent le mode de production scientifique où les thèmes de recherche sont définis par les seuls chercheurs et le mode où les utilisateurs de la recherche sont impliqués dans la définition des thèmes et la formulation des problèmes.

-« l'autonomie et l'hétéronomie » : les questions sur la liberté du chercheur, la liberté d'initiative dans la conduite de la recherche font ici miroir.

-« le marché et la gratuité »: les enjeux sur le financement de la recherche dans une perspective de recherche de profit (exemple: les résultats brevetables) sont ici mis en perspective. Le financement joue un rôle important dans la stimulation de la production du savoir (Chasseriaux, 2004).

-« le territorial et le global »: les questions d'alliances, d'intégration entre structures de recherche ou laboratoires d'un même site ou d'un plus large bassin sont ici prises en compte.

-« le long terme et le court terme » : les questions de vision et de réflexions sur les priorités de recherche sont ici posées.

Tenant compte de tout ce qui précède et du fait que l'étiquette «stratégique » est pertinente dès lors que des actions et décisions sont associées à un degré d'irréversibilité ou cristallisent des enjeux ou encore exercent un effet sur un système (Roland et Tugrul, 1991), nous considérons que le management stratégique de la recherche renvoie aux décisions et actions qui visent à orienter l'activité de recherche vers des objectifs définis dans un horizon de moyen et long terme. Ces décisions et actions consistent en des arbitrages entre le «territorial et le global », la « fermeture et l'ouverture », l'« autonomie et l'hétéronomie », ou encore «le marché et la gratuité ».

\section{Eclairage sur le concept de dysfonctionnement emprunté dans cette réflexion.}

Le concept de dysfonctionnement est approché dans l'étude en se référant aux éclairages des chercheurs de l'ISEOR ${ }^{27}$.

Tableau 1. Le dysfonctionnement vu par les auteurs

\begin{tabular}{|l|l|l|}
\hline Auteurs & $\begin{array}{l}\text { Type de } \\
\text { contribution }\end{array}$ & Regard sur le dysfonctionnement \\
\hline $\begin{array}{l}\text { Martinet et Savall } \\
(1978)\end{array}$ & $\begin{array}{l}\text { Contribution } \\
\text { théorique }\end{array}$ & $\begin{array}{l}\text { Est considéré comme dysfonctionnement un } \\
\text { écart ressenti par rapport au fonctionnement } \\
\text { attendu dans l'organisation, fonctionnement } \\
\text { référencé selon la norme habituellement } \\
\text { acceptée. }\end{array}$ \\
\hline
\end{tabular}

${ }^{27}$ Institut de Socio-économie des Entreprises et des Organisations. L'ISEOR est un centre de recherche autonome associé à l'université Jean Moulin et, jusqu'en 2007, à l'Ecole de Management de Lyon. 


\begin{tabular}{|c|c|c|}
\hline $\begin{array}{l}\text { Nader et Peretti } \\
(2007)\end{array}$ & $\begin{array}{l}\text { Etude } \\
\text { exploratoire }\end{array}$ & $\begin{array}{l}\text { Les dysfonctionnements } \text { représentent des } \\
\text { anomalies, perturbations, conflits, tensions } \\
\text { subis par l'entreprise. }\end{array}$ \\
\hline $\begin{array}{l}\text { Santos et Mousli } \\
(2009)\end{array}$ & $\begin{array}{l}\text { Recherche } \\
\text { intervention }\end{array}$ & $\begin{array}{l}\text { Est perçu comme dysfonctionnement tout écart } \\
\text { entre fonctionnement attendu et fonctionnement } \\
\text { réel. }\end{array}$ \\
\hline Bouyoud, (2007) & $\begin{array}{l}\text { Recherche } \\
\text { intervention }\end{array}$ & $\begin{array}{l}\text { Le dysfonctionnement traduit un écart entre le } \\
\text { fonctionnement souhaité et le fonctionnement } \\
\text { constaté. }\end{array}$ \\
\hline Cappelleti (2006) & $\begin{array}{l}\text { Recherche } \\
\text { intervention }\end{array}$ & $\begin{array}{llll}\text { un dysfonctionnent figure } & \text { un état } \\
\text { d'insatisfaction } & & & \\
\end{array}$ \\
\hline $\begin{array}{l}\text { Cappelletti et } \\
\text { Levieux (2010) }\end{array}$ & Etude de cas & $\begin{array}{l}\text { Les dysfonctionnements perturbent les acteurs } \\
\text { dans leurs équipes, leurs lieux de travail. }\end{array}$ \\
\hline $\begin{array}{l}\text { Cristallini et } \\
\text { Goter-Grivot } \\
(2012)\end{array}$ & $\begin{array}{l}\text { Recherche } \\
\text { intervention }\end{array}$ & $\begin{array}{l}\text { Les entretiens d'écoute permettent aux } \\
\text { personnes interviewées de s'exprimer sur les } \\
\text { dysfonctionnements qu'elles rencontrent dans } \\
\text { leur travail. Ces dysfonctionnements sont des } \\
\text { symptômes de pathologies qui grèvent } \\
\text { l'efficacité du fonctionnement. }\end{array}$ \\
\hline Rymeyko (2007) & $\begin{array}{l}\text { Recherche } \\
\text { intervention }\end{array}$ & $\begin{array}{l}\text { Les dysfonctionnements sont identifiés dans les } \\
\text { pratiques des responsables à partir du } \\
\text { vocabulaire expressif qu'ils utilisent pour en } \\
\text { rendre compte lors d'entretiens. }\end{array}$ \\
\hline $\begin{array}{l}\text { Martinez Vazquez } \\
(2005)\end{array}$ & $\begin{array}{l}\text { Recherche } \\
\text { intervention }\end{array}$ & $\begin{array}{l}\text { Les dysfonctionnements peuvent concerner le } \\
\text { niveau opérationnel et le niveau stratégique. }\end{array}$ \\
\hline $\begin{array}{l}\text { Barel et Fremeaux } \\
(2011)\end{array}$ & Etude de cas & $\begin{array}{l}\text { Les dysfonctionnements détériorent la } \\
\text { performance. }\end{array}$ \\
\hline Pierre (2010) & $\begin{array}{l}\text { Recherche } \\
\text { intervention }\end{array}$ & $\begin{array}{l}\text { Au cours des entretiens sont exprimés les } \\
\text { dysfonctionnements ressentis par les acteurs. } \\
\text { Ces dysfonctionnements brident la capacité des } \\
\text { organisations à se transformer. }\end{array}$ \\
\hline
\end{tabular}

Source : Revue de la littérature.

Lorsqu'on examine les données du tableau ci-avant, nous pouvons retenir deux principaux enseignements :

- les dysfonctionnements perturbent la vie de l'organisation, ils ne sont pas compatibles avec la recherche de l'efficacité optimale et l'exploitation efficiente des ressources matérielles et humaines ;

- les dysfonctionnements manifestent un état d'insatisfaction ressenti par les acteurs au sein de leur organisation. Cet état d'insatisfaction figure tout écart entre un état souhaité, c'est à dire perçu comme normal par les acteurs de l'organisation, et l'état réellement vécu par ces derniers. 


\section{Démarche méthodologique}

Ce travail se prête bien au genre étude de cas. De Laat et Barré (2007, p. 36) indiquent fort bien que « chaque Institution de recherche a son équation propre en termes de missions, positionnement, histoire, mais aussi taille, ressources, types de personnel etc. Sa stratégie et son management lui sont donc spécifiques.». Lorsqu'on veut appréhender les spécificités d'un phénomène, que ce phénomène concerne des questions de management ou non, l'étude de cas demeure appropriée (Stake, 1998).

L'UCAD choisie comme terrain d'enquête (l'UCAD est un terrain plus accessible ${ }^{28}$ que les autres universités publiques du Sénégal situées toutes hors de la capitale (Dakar)), nous avons appliqué une stratégie d'échantillonnage à variation maximale. Les acteurs enquêtés n'ont pas les mêmes qualités (cf. tableau infra). L'exercice de collecte des données a couvert un panel de 07 responsables qui, de par les positions centrales qu'elles occupent dans le système de recherche de l'UCAD, constituent des acteurs clés auprès de qui les pratiques de management de la recherche au sein de l'institution peuvent être saisies ou appréhendées. Pratiques dont ces responsables incarnent «l'esprit et le corps ».

Le tableau ci-après présente les caractéristiques de ces enquêtés. L'anonymat est préservé en désignant chaque responsable par le code Ri où $\mathrm{R}=$ Responsable et $\mathrm{i}=$ numéro d'ordre dans le déroulement des enquêtes. Le guide d'entretien utilisé est semi-structuré. Il a permis d'entretenir les discussions avec les enquêtés autour de six principaux points :

- la place de la recherche universitaire dans les politiques nationales ;

- les défis de la recherche universitaire sur le moyen et long terme ;

- l'environnement de la recherche à l'UCAD ;

- les questions d'orientation, de définition de priorités de recherche ;

- les questions d'impact de la recherche et de valorisation ;

- et les questions d'animation de la recherche universitaire.

L'ordre de discussion sur ces différents points n'était pas imposé aux enquêtés mais déterminé par nous-mêmes en fonction de l'enchaînement des idées propres aux répondants. Cette flexibilité permettait de vérifier la cohérence des informations communiquées par les enquêtés.

Ont servi de données de seconde main : le Plan stratégique de l'UCAD, les textes et lois sur l'organisation de la recherche à l'UCAD et les documents en rapport avec les stratégies nationales de développement au Sénégal. Ces sources sont utiles pour la triangulation (Hlady Rispal, 2002).

${ }^{28}$ Notre résidence se situe à quelques $9 \mathrm{KM}$ de l’UCAD 


\begin{tabular}{|c|c|c|c|c|}
\hline \multirow{2}{*}{ code } & \multirow{2}{*}{ Qualités des personnes enquêtées } & \multicolumn{2}{|r|}{ Entretiens } & \multirow[t]{2}{*}{ Informations complémentaires } \\
\hline & & Nombre & Durée & \\
\hline $\mathrm{R} 1$ & $\begin{array}{l}\text { Haut responsable au Ministère de l'Enseignement } \\
\text { supérieur et de la recherche du Sénégal. }\end{array}$ & 1 & $\begin{array}{l}\text { Durée totale : } 58 \mathrm{mn} \\
48 \mathrm{~s} \text {. }\end{array}$ & $\begin{array}{l}\text { Les tentatives de contact en vue d'une seconde interview auprès de R1 n'ont pas } \\
\text { eu de succès. Néanmoins, nous avons eu la validation par e-mail de la première } \\
\text { entrevue retranscrite in extenso et envoyée à R1 }\end{array}$ \\
\hline R2 & $\begin{array}{l}\text { *Responsable Ecole doctorale « Sciences de la Vie, de } \\
\text { la Santé et de l'Environnement » à l'UCAD. } \\
\text { *Directeur au laboratoire de physiologie, d'explorations } \\
\text { fonctionnelles physiologiques et de biologie appliquée } \\
\text { aux sports. }\end{array}$ & 3 & $\begin{array}{l}\text { Mini : } 27 \mathrm{mn} 20 \mathrm{~s} \\
\text { Maxi : } 51 \mathrm{mn} 44 \mathrm{~s}\end{array}$ & \multirow{6}{*}{$\begin{array}{l}\text { Pour tous les autres enquêtés ( } \mathrm{R} 2 \text { à } \mathrm{R} 7 \text { ), la règle de la saturation empirique est } \\
\text { observée. La collecte est arrêtée lorsque le répondant ne révélait plus rien de } \\
\text { nouveau sur l'objet. } \\
\text { La saturation a coïncidé avec le troisième entretien pour R2, R3 et R7. Elle est } \\
\text { atteinte à l'issue du second entretien pour R4, R5 et R6. }\end{array}$} \\
\hline R3 & $\begin{array}{l}\text { Responsable au Laboratoire Chimie de Coordination } \\
\text { Organique à l'UCAD. } \\
\text { Responsable master de recherche à la Faculté des } \\
\text { Sciences et Techniques /UCAD. }\end{array}$ & 3 & $\begin{array}{l}\text { Mini : 39mn 01s } \\
\text { Maxi : 01h10mn 49s }\end{array}$ & \\
\hline R4 & $\begin{array}{l}\text { *Responsable Groupe de Recherche en Physique du } \\
\text { Solide et en Sciences des Matériaux à l'UCAD. } \\
\text { *Directeur de recherche au laboratoire centrale de } \\
\text { Hitarchie à Tokyo pendant des années, } \\
\text { *Directeur du centre africain des lasers à Pretoria pendant } \\
\text { des années }\end{array}$ & 2 & $\begin{array}{l}\text { Mini : } 29 \mathrm{mn} 33 \mathrm{~s} \\
\text { Maxi : } 43 \mathrm{mn} 46 \mathrm{~s}\end{array}$ & \\
\hline R5 & $\begin{array}{l}\text { *Responsable Ecole doctorale Mathématiques- } \\
\text { Informatiques / UCAD } \\
\text { *Responsable laboratoire d'Algèbre, de Cryptologie, de } \\
\text { Géométrie Algébrique et Applications / UCAD }\end{array}$ & 2 & $\begin{array}{l}\text { Mini : } 43 \mathrm{mn} 43 \mathrm{~s} \\
\text { Maxi : } 01 \mathrm{~h} 10 \mathrm{mn} \\
04 \mathrm{~s}\end{array}$ & \\
\hline R6 & $\begin{array}{l}\text { *Responsable Ecole doctorale ARCIV : "Arts, Cultures, } \\
\text { Civilisations" / UCAD } \\
\text { *Responsable laboratoire Sociolinguistique, Linguistique } \\
\text { et Didactique des Langues en Afrique/ UCAD }\end{array}$ & 2 & $\begin{array}{l}\text { Mini : 45mn 40s } \\
\text { Maxi : } 01 \mathrm{~h} 17 \mathrm{mn} \\
24 \mathrm{~s}\end{array}$ & \\
\hline R7 & $\begin{array}{l}\text { *Responsable à la Direction de la recherche de l'UCAD } \\
\text { *Membre du conseil scientifique de l'UCAD } \\
\text { *Responsable laboratoire Parasitologie générale / UCAD }\end{array}$ & 3 & $\begin{array}{l}\text { Mini : } 30 \mathrm{mn} 09 \mathrm{~s} \\
\text { Maxi : } 56 \mathrm{mn} 34 \mathrm{~s}\end{array}$ & \\
\hline
\end{tabular}

Tableau 1 : Entretiens et qualités des enquêtés. 
Le matériau recueilli est exploité grâce à l'analyse de contenu en s'appuyant sur le logiciel Tropes (version 8.4).Trois phases ont marqué la codification.

Phase 1: l'étiquetage thématique

Dans cette phase, les passages d'entrevues qui renvoient à des significations différentes sont repérés par une étiquette à l'aide d' ' un mot ou une expression et non par une phrase complète » (Lannoy, 2012 p. 6).

Tableau 2. Mouture d'étiquettes générées à partir du corpus.

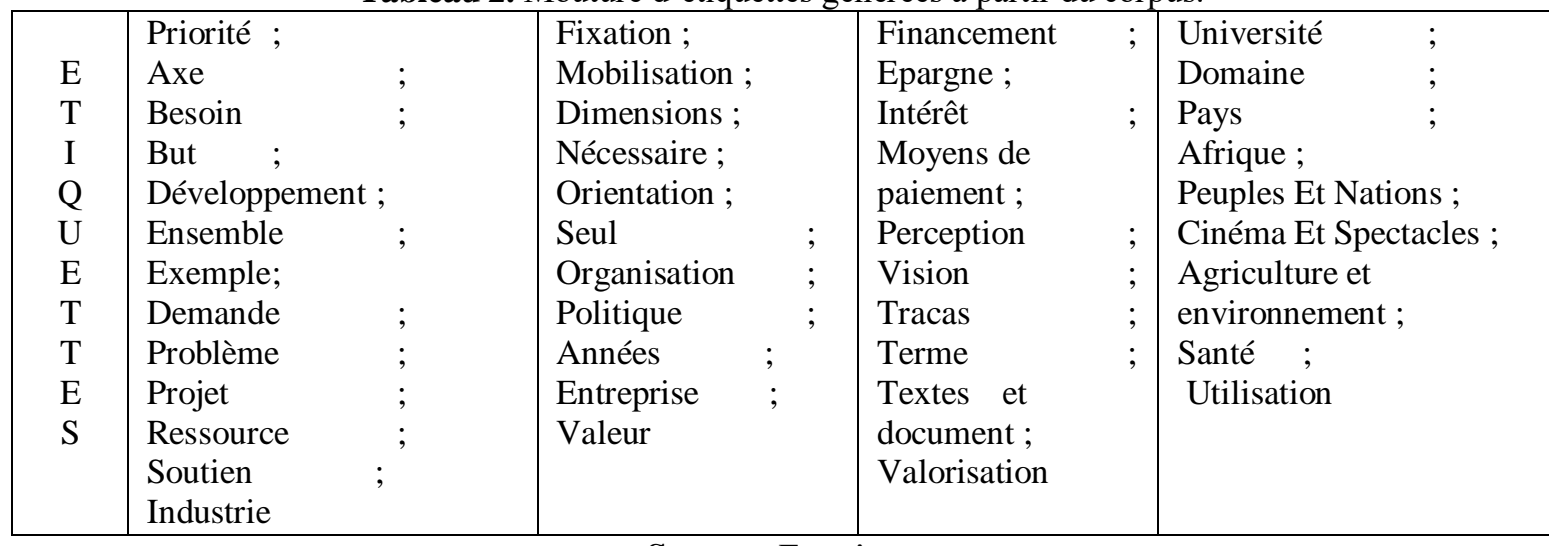

Source : Enquêtes.

Les étiquettes générées par le logiciel pendant cette phase peuvent requérir un toilettage avant d'être retenu définitivement. En effet, le dictionnaire du logiciel utilisé (en l'occurrence Tropes) peut proposer des étiquettes que le chercheur devra, dans une phase dite de consolidation, systématiquement :

- éliminer si elles se révèlent impertinentes par rapport aux objectifs de l'étude ;

- regrouper lorsqu'elles sont désignées différemment par le logiciel alors qu'elles correspondent à des passages du matériau au sens identique ;

- reformuler par des termes plus collés aux passages qui les supportent que ce que propose le logiciel ;

- épurer pour s'assurer que les étiquettes désignées par le logiciel sont irréductibles d'un point de vue sémantique.

L'économie de ces différentes opérations conduites en conséquence est rendue par le tableau infra. 
Tableau 3. Dynamique de consolidation des étiquettes

\begin{tabular}{|c|c|c|c|}
\hline $\begin{array}{l}\text { Etiquettes } \\
\text { éliminées }\end{array}$ & Etiquettes regroupées & Etiquettes reformulées. & Etiquettes épurées \\
\hline $\begin{array}{l}\text { Seul ; } \\
\text { Ensemble ; } \\
\text { Cinéma et } \\
\text { spectacle ; } \\
\text { Moyen de } \\
\text { paiement ; } \\
\text { Année }\end{array}$ & 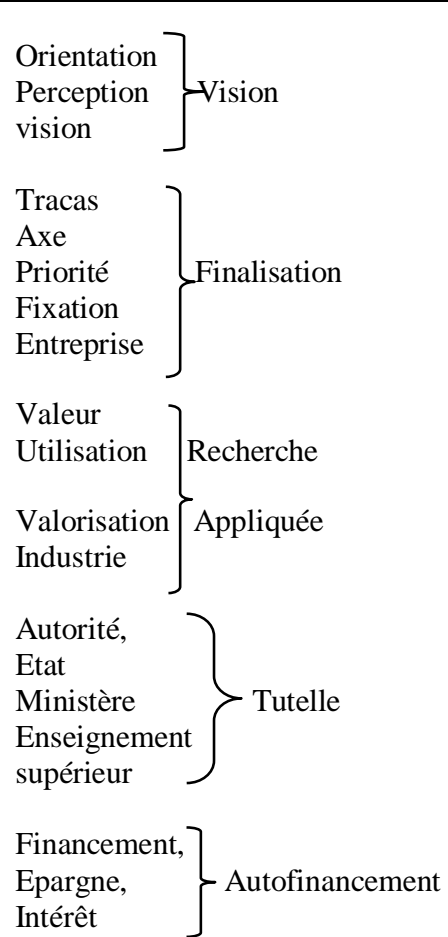 & $\begin{array}{l}\text { But } \longrightarrow \text { Objectif } \\
\text { Demande } \rightarrow \text { financement } \\
\text { Extérieur }\end{array}$ & $\begin{array}{l}\text { Projet }\left\{\begin{array}{l}\text { Financement extérieur } \\
\text { Finalisation }\end{array}\right. \\
\text { Problème }\left\{\begin{array}{l}\text { Finalisation } \\
\text { Organisation }\end{array}\right. \\
\text { Afrique/Pays }\left\{\begin{array}{l}\text { Financement extérieur } \\
\text { Développement }\end{array}\right. \\
\text { Domaine }\left\{\begin{array}{l}\text { Agriculture et environnement } \\
\text { Santé }\left\{\begin{array}{l}\text { Vision } \\
\text { Finalisation }\end{array}\right.\end{array}\right. \\
\text { Soutien / Ressource }\left\{\begin{array}{l}\text { Autofinancement } \\
\text { Allocation de } \\
\text { Ressource }\end{array}\right. \\
\text { Textes et } \\
\text { documents }\left\{\begin{array}{l}\text { Définition des objectifs } \\
\text { Choix des moyens financiers }\end{array}\right.\end{array}$ \\
\hline
\end{tabular}

Source : Enquêtes. 
Les opérations réalisées au niveau du tableau ci-dessus ont permis d'adapter, de consolider les références thématiques proposées par Tropes à l'aide de son propre dictionnaire. La phase 3 ou phase de classification des thèmes peut alors débuter.

Phase 3 : classification thématique

Les étiquettes définitivement retenues lors de la phase consolidation (Phase 2 ci-avant) sont reprises ici pour la production d'un thésaurus arborescent. Ce thésaurus, à l'image de la métaphore de Savall et Zardet (2004), comprend un tronc (une méta-catégorie), des branches porteuses constituées de catégories, de petites branches correspondant à des souscatégories et où les feuilles ou fleurs sont les thèmes identifiés à partir des citations, expressions contenues dans les entretiens (tableau 4).

Tableau 4. Classification hiérarchique

\begin{tabular}{|c|c|c|c|}
\hline Thèmes & Sous-catégories & Catégories & Méta-catégorie \\
\hline $\begin{array}{l}\text { Agriculture et } \\
\text { environnement }\end{array}$ & \multirow[t]{3}{*}{ Domaine prioritaire } & \multirow{8}{*}{ Prospective } & \multirow{14}{*}{$\begin{array}{l}\text { Management } \\
\text { stratégique }\end{array}$} \\
\hline Santé & & & \\
\hline Développement & & & \\
\hline Attentes des populations & \multirow{5}{*}{ Finalisation } & & \\
\hline Vision & & & \\
\hline Politique & & & \\
\hline Collaboration & & & \\
\hline Rôle de la tutelle & & & \\
\hline Objectif & \multirow{6}{*}{$\begin{array}{l}\text { Engagement de } \\
\text { ressource }\end{array}$} & \multirow{6}{*}{ Stratégie } & \\
\hline Terme & & & \\
\hline Autofinancement & & & \\
\hline Financement extérieur & & & \\
\hline Financement national & & & \\
\hline Recherche appliquée & & & \\
\hline
\end{tabular}

Source : Par l'auteur

Outre les données d'entretien, nous avons eu recours à l'observation non participante et exploité les textes officiels sur l'UCAD en rapport notamment avec ses activités de recherche. Aussi, nous avons exploité des textes du site officiel du gouvernement du Sénégal en rapport avec les stratégies nationales de développement.

\section{Présentation des Résultats}

Pour rappel, les dysfonctionnements correspondent aux faiblesses dont sont témoins les acteurs au sein du système où ils sont parties prenantes. D'une manière plus opératoire, un dysfonctionnement correspond à l'écart entre un état souhaité et la situation actuellement vécue. Les dysfonctionnements sont examinés au niveau des catégories construites antérieurement (cf. supra). Ces 
catégories se rapportent aux composantes «stratégie » et «prospective » dont les occurrences ont été déterminées à l'aide du logiciel Tropes V8.

Tableau 5. Occurrences des dimensions du MSR

\begin{tabular}{|l|c|c|c|}
\hline Sous-catégories & Fréquences & Catégories & Méta-catégorie \\
\hline Finalisation & 26 & Prospective & \multirow{3}{*}{ Management stratégique } \\
\cline { 1 - 2 } Domaine prioritaire & 10 & $(36)$ & Stratégie \\
\cline { 1 - 2 } Objectif & 21 & $(36)$ & \\
\cline { 1 - 2 } Engagement ressource & 15 & (72) \\
\cline { 1 - 2 }
\end{tabular}

Source : données d'enquêtes.

Au regard du tableau, nous retrouvons deux composantes principales dans le MSR.

$\checkmark$ La composante prospective

L'analyse horizontale faite sur le corpus révèle que la prospective dans les politiques publiques ne s'attarde pas sur comment bâtir un modèle de recherche universitaire performant au service des questions d'intérêt national. Plus dans le détail, des questions d'envergures de type quelles recherches privilégiées dans les domaines identifiés comme prioritaire au niveau national ? Pour quels impacts sur les citoyens et les générations à venir ? Le tout avec quel «système de contractualisation viable » entre la tutelle (représentée par l'Etat du Sénégal) et l'université qui abrite un potentiel non négligeable de structures de recherche ? constituent un pan orphelin dans la prospective. A la lumière du terrain, l'Etat du Sénégal ressort comme un acteur principal sur chacun de ces registres : "Il faut que la plus haute autorité définisse les intérêts du pays. Sur quels sujets les chercheurs doivent être mobilisés pour qu'il y ait un input important pour le pays. Sinon chacun ira dans sa direction (....). » (Martèle le responsable R3).

Toutefois, les données recueillies ne permettent pas d'assimiler l'Etat à celui qui doit être « le seul gardien de la vision prospective ». L'université, au travers de ses acteurs et des structures de recherche, est vigoureusement interpellée. Sa faible collaboration avec l'autorité de tutelle dessert les composantes «finalisations » et «domaine prioritaire » (deux facteurs clé dans la prospective).

«La recherche universitaire doit apporter des solutions aux problèmes de développement que nous avons. Par exemple nous avons un problème de semence, que les chercheurs se remettent à l'œuvre pour proposer des solutions! » R1

"Normalement il devrait y avoir des réflexions dans les écoles doctorales pour voir quels sont les thèmes qui sont porteurs de développement à partir des politiques définies au niveau national. Chaque école doctorale devrait voir l'action de recherche qui peut être menée dans ce domaine-là. Mais à ma connaissance jusqu'à présent ce n'est pas ça qui est fait. C'est plutôt une structure administrative. » $\mathbf{R 5}$ 
En convoquant les textes des politiques de développement au niveau national, l'on note que la plus grande occurrence de la dimension «finalisation» par rapport à l'occurrence de la dimension «domaine prioritaire » (cf. tableau 5) ne relève pas de l'artéfact. En effet, dans ces documents, nous remarquons que si la prospective est convaincante sur l'aspect détermination des domaines prioritaires ayant un fort potentiel de développement, l'aspect finalisation (sur le devenir de la recherche universitaire, les choix des domaines de recherche, les rôles à attendre des universitaires etc.) l'est moins. A ce propos, lorsqu'on considère la Stratégie de Croissance Accélérée (SCA) ou encore le Plan Sénégal Emergent (PSE), deux documents qui ont respectivement marqué la première et la seconde alternance politique au Sénégal, la réflexion est riche de l'identification de plusieurs domaines à fort potentiel de développement. Toutefois, la place de la recherche, notamment son rôle dans le développement, semble être reléguée au second rang dans ces documents. L'article 2 de la loi d'orientation sur la SCA, centré sur les termes considérés comme essentiels dans la mise en œuvre, délimite le cadre conceptuel du texte de loi. Cette partie va jusqu'à définir l'entreprise ${ }^{29}$ dans le cas de ladite stratégie ainsi que ces différents types qui sont ciblés en passant sous silence les structures de recherche convoquées par endroit suivant les termes « institutions de recherche » (article 24) et « centre de recherche » (article 26).

Dans le chapitre du PSE portant «enseignement supérieur et recherche », la vision prospective autour de la recherche reste très timide, elle arrête des choix de domaines mais réduit leur portée en ne mettant la centralité que sur des caps d'effectifs :

«Dans le domaine de l'enseignement supérieur et de la recherche, le Gouvernement accordera la priorité aux sciences et à la technologie, aux sciences de l'ingénieur et à la recherche appliquée afin :

- d'atteindre 50\% de bacheliers scientifiques,

- de porter à 50\% la part des effectifs de l'enseignement supérieur poursuivant une formation professionnelle,

- de réduire la concentration des établissements sur la côte atlantique,

- et d'atteindre un taux de 20\% à 30\% d'étrangers dans les effectifs du supérieur en $2025^{30} »$

Dans le verbatim ci-dessus, la dimension finalisation néglige le troisième cycle alors que c'est celui-ci qui produit les chercheurs en herbe pour la société.

${ }^{29}$ Dans la présente loi, sont visées les entreprises de toutes natures et de toutes catégories : exploitations individuelles, micro-entreprises, petites et moyennes entreprises, grandes entreprises, multinationales » (Article 2, Loi d'orientation)

${ }^{30}$ Cf. PSE (2014-2015) à la page 79. 
Si dans le MSR la composante «finalisation» tient insuffisamment compte des défis qui interpellent le secteur de la recherche et les rôles que ce dernier doit jouer dans les politiques de développement c'est parce qu', entre « enseignement » et « recherche », l'un mobilise plus l' «énergie » de la tutelle au détriment de l'autre. Plus clairement, le traitement des urgences au niveau de l'enseignement supérieur est l'objet qui préoccupe plus la tutelle. «Il faut qu'on ait un ministère de la recherche digne du nom. Ce ministère-là ne doit s'occuper qu'exclusivement que de recherche. L'enseignement supérieur crée des problèmes maintenant si tu mets ça avec la recherche qu'est-ce que ça va donner? Sachant que ce ne sont pas les chercheurs qui vont casser des bus ....donc les responsables de ce ministère vont s'occuper de quoi ? De ceux qui cassent au détriment de la recherche! Donc y a lieu de séparer ces deux ministères. Le ministère de la recherche doit être traité comme un ministère de souveraineté. On doit traiter ce ministère comme la Défense. Que ce ministère ne soit pas soumis aux aléas de la vie politique. " (Martèle le responsable $\mathrm{R} 3$ ).

Les données portant création, organisation et fonctionnement de l'ANAQ $^{31}$ et le texte officiel ${ }^{32}$ portant création de l'Université virtuelle du Sénégal (UVS) montrent respectivement que les priorités sont au niveau de la qualité et de la maîtrise des effectifs :

"le Ministère de l'Enseignement supérieur et de la Recherche s'est fixé, pour les années à venir, un certain nombre de priorités dont l'amélioration de la qualité des formations et l'efficacité des établissements. »

- «Le sous-secteur de l'Enseignement Supérieur et de la Recherche fait face à un réel problème d'accès. Les projections sur les effectifs de bacheliers dans la période 2012-2022 donnent un taux d'accroissement très important qui ne pourra être résorbé par les projets de construction de nouveaux établissements d'enseignement supérieur publics. (....)».

Le point qui suit analyse la composante «stratégie» dans le MSR.

\section{$\checkmark$ La composante stratégie}

Elle est examinée en s'appuyant sur les deux sous catégories suivantes ressorties dans l'analyse thématique : le financement et le choix des objectifs (cf. tableau 5 supra).

Le premier constat qui émerge du matériau a trait au besoin d'avoir des objectifs de recherche partagés au niveau national (principe d'énergie). Le second constat a trait à l'importance de la mobilisation des moyens pour accompagner ces objectifs (principe d'instrumentation). La fonction d'instrumentation confère au financement son caractère stratégique. Ce rôle

\footnotetext{
${ }^{31}$ ANAQ : Autorité Nationale d'Assurance Qualité

${ }^{32}$ Décret n$^{\circ}$ 2013-1294 du 23 septembre 2013
} 
d'instrumentation se mesure au travers de son influence sur l'orientation de la recherche.

"L'autorité doit mettre en place les moyens pour financer les chercheurs (....). Sinon chacun ira dans sa direction et les directions où nous allons souvent épousent plus les intérêts de ceux qui financent que les intérêts de notre pays. » R3.

«S'il y a recherche c'est impulsé de l'extérieur par les bailleurs qui proposent des projets de recherche. Et l'université en profite car c'est le seul moyen pour lui de produire. Le problème que cela pose si nous restons dépendant de ces bailleurs, c'est qu'ils nous imposent des objectifs qui ne rencontrent pas forcément nos préoccupations.» R4.

«Dans mon domaine les opportunités de financement sont très marginales. Pourtant, il existe beaucoup de possibilités de recherche nécessaires (exemple: la grammatisation des langues nationales, la production de dictionnaires, dictionnaires sérères, wolof etc.) où l'initiative individuelle ne suffit pas. Quelle est la part du budget pour promouvoir ces langues nationales? Alors qu'on a de jeunes chercheurs qu'on peut encadrer dans ces thématiques.» R6.

Au regard de ces verbatim, le financement extérieur ressort comme une source qui ne va pas forcément dans le sens des préoccupations au niveau national. Les enjeux sur le financement dépassent leur impact sur le choix des thèmes. C'est aussi des enjeux de valorisation économique des résultats de la recherche. Le responsable (R7), acteur central à la Direction de la recherche, confie: «Y a des enseignants-chercheurs qui ont déposé leur brevet à l'UCAD. Mais ils se battent chacun pour pouvoir le défendre. L'UCAD n'a pas les moyens pour défendre les brevets. Il y a des cabinets spécialisés pour faire la promotion de ces brevets et il faut les payer ».

Au demeurant, l'idée qui émerge du verbatim ci-dessus (tiré des entretiens avec R7, cf. ci-avant) faisant état d'un déficit d'assistance des chercheurs dépositaires de brevet trouve un écho dans ces données primaires :

«Nous concentrons plus de temps à la recherche fondamentale. On perd trop de temps pour dérouler nos protocoles. Certaines manipulations nous ne pouvons les faire que dans le cadre de la coopération. Si tu dépends entièrement des bailleurs cela pose problème. Les clauses profitent aux bailleurs $\gg \mathrm{R} 2$

«Nous avons 4 brevets mais aucun n'est passé à la phase pilote. Nous $n$ 'avons pas les moyens juridiques pour nous protéger. On dit oui protection intellectuelle c'est bien beau mais si le gars viole ton idée et toi tu n'as pas $d$ 'argent pour l'attaquer. Le cabinet d'avocat ou le cabinet de conseil il faut le payer et l'université ne t'offre même pas l'espace pour s'exprimer sur ce plan ». R3 


\section{Discussion des résultats}

L'un des enseignements très saisissants, révélés par l'exploitation des données empiriques, est que la réflexion prospective est aussi importante que la stratégie. Ces deux composantes enregistrent la même occurrence dans le matériau (cf. Tableau 5 dans le point sur les résultats). Toute chose égale par ailleurs et en termes de fréquence, elles ont la même part d'attention chez les responsables enquêtés lorsqu'on considère l'ensemble des données d'entretiens colligées. On peut rapprocher ce résultat des remarques de Godet (2004) qui considère la prospective et la stratégie comme deux domaines d'égale importance où chacun appelle l'autre.

Un autre point de comparaison est consécutif aux remarques de Mintzberg (2004) qui indique que le manager préfère l'action immédiate à l'action différée. Il est absorbé par les sollicitations de l'organisation et de son environnement (courrier, téléphone, discussion etc.). De nos analyses, il est ressorti que l'autorité de tutelle (l'Etat) est plus préoccupée par la gestion des urgences dans l'enseignement supérieur que par les questions de prospective pour la recherche universitaire. Autant ce résultat va dans le sens des travaux de Mintzberg, autant également il peut être mis en parallèle avec les remarques de Sock (2006). Ce dernier ne manque pas de préciser dans ses travaux qu'en Afrique francophone, le potentiel des universités n'est pas suffisamment mis à profit dans les stratégies de développement socio-économiques.

Les résultats montrent que le troisième cycle est relégué au second rang alors que c'est ce cycle qui produit les chercheurs en herbe pour la société. Sawyer (2004) précise à ce sujet que la valorisation des études de troisième cycle est l'un des défis que l'Afrique doit relever pour répondre aux exigences de l'économie du savoir. Dans le même sens, Altbach (2007, p. 130) souligne que lorsqu'on soutient le troisième cycle, on fait profiter à la recherche la grande motivation des étudiants en doctorat qui, en retour, «tirent parti de leur participation directe à des travaux de pointe ».

Un regard rétrospectif autorise un rapprochement entre les résultats de l'étude et ceux de Plane (1994). Ce dernier a montré que les acteurs hiérarchiques adoptent une stratégie d'expulsion et d'attribution de dysfonctionnements à d'autres acteurs. Nos entretiens ont révélé ce phénomène. Au travers des données collectées auprès des responsables R1, $\mathrm{R} 2$ et R7 (pour ne citer que ces derniers), les indices qui illustrent le mieux ce phénomène de «passe d'armes » sus évoqué sont rendus dans l'encadré 1 ciavant. 
Encadré 1 : « Stratégie d'attribution, d'expulsion de dysfonctionnements ».

R1 qui est au ministère « charge » l'UCAD avec volubilité. Le langage co verbal dans les verbatim est très évocateur :

- «Nous avons un problème de semence. Que les chercheurs se remettent à l'œuvre pour proposer des solutions!";

- "Il faut que tous les programmes de recherche puissent être identifiés à l'UCAD ainsi que les acteurs. (....) pour que si on met les pieds à l'UCAD et qu'on serait intéressé par une question bien précise qu'on puisse identifier de manière efficace l'interlocuteur approprié pour avoir des réponses sur ces questions. »

Au tour de $\mathbf{R 2}$ (directeur d'école doctorale à l'UCAD) et $\mathbf{R 7}$ (responsable à la direction de la recherche de l'UCAD) de renvoyer l'ascenseur à la tutelle :

- "Si l'Etat définit ses priorités de recherche, se donne les moyens pour régler ses priorités de recherche, on peut maintenant exiger au chercheur des résultats dans ce sens. Mais tant que cela n'est pas effectif, on ne peut rien exiger du chercheur. " (R2) ;

- "Il n'existe pas de document fixant les objectifs de recherche au niveau national. Dans ce document on doit fixer des indicateurs pour évaluer les objectifs. Dans le même document il faut définir les moyens. A partir de là les institutions de recherche pourront être évalués. Le Fonds qui serait mis en place pourrait être alimenté (...) à partir du budget de l'Etat. »(R7)

Source : Données d'enquête.

Les modalisations dans les deux verbatim (R2) et (R7) ci-avant sont parlantes. Elles forcent le trait sur des manquements qui interpellent directement la tutelle et par la même occasion atténuent (pour ainsi dire) la responsabilité des chercheurs. Cette tendance des enquêtés consistant à se rejeter des dysfonctionnements répond d'un souci de défendre chacun une façade acceptable. Plane (1994, p.104), dans ses travaux, commente ce phénomène en ces termes : «nous avons remarqué que certains acteurs semblent motivés par la préservation d'une façade sociale qu'ils jugent acceptable. Nous pensons que ces personnes veulent, consciemment ou inconsciemment, se protéger contre certains dysfonctionnements auxquels les chercheurs pourraient les associer. ». Dans nos résultats, cette protection se traduit par une tendance des acteurs à plutôt montrer (voire valoriser) ce qu'ils font de positifs ou envisagent de faire (cf. encadré 2 ci-après). 
Encadré 2. «Préservation d'une façade acceptable »

R1 s'enorgueillit d'initiatives qui ne sont pas encore opérationnalisées : «le Fonds d'Impulsion de Recherche Scientifique et Technique sera transformé en Fonds National de Recherche et d'Innovation pour mieux relier les résultats de la recherche aux questions d'innovation, pour promouvoir le transfert des connaissances vers la production et l'utilisation de la connaissance »

$\mathbf{R 2}$ n'est pas en reste :

« $\mathrm{A} u$ niveau de l'école doctorale, nous avions identifié la coopération française parmi les voies possibles pour disposer d'équipement. L'objectif était au moins d'avoir des équipements dans le domaine de l'expression des protéines. Les démarches entreprises à cette fin étaient, au départ, incertaines. Finalement nous avons obtenu 10 millions sur un besoin de 100 millions. Mais que faire, il faut passer par la coopération. »

R3 s'auto glorifie :

«On fouille tout le temps sur internet, on regarde les acteurs qui financent la recherche. $Y$ a quand même des mécènes, des organisations comme l'AUF ${ }^{33}$, le $C R D I^{34}$ etc. qui financent la recherche et chaque fois qu'ils font un appel nous soumissionnons »; "Si on vous montre où on élabore nos résultats vous serez ahuri. Nos propres collègues espagnols qui nous rendent visite se demandent comment nous faisons pour sortir tous nos résultats! Même y a des étudiants qui viennent adhérer à notre labo sans qu'on ne le leur demande. ")

R7 (à l'image de R1) s'enorgueillit du projet d'utilisation de softwares au service de la recherche à l'UCAD :

« l'UCAD compte mettre en ouvre un logiciel avec l'appui de l'AUA en vue de rendre visible son activité de recherche »

\section{Source : Données d'enquête.}

En remarque, les révélations notées à l'occasion des entretiens dysfonctionnels confèrent une certaine validité externe (ou une généralisabilité selon le terme préféré de certains auteurs en recherche qualitative qui considèrent ce vocable comme étant plus approprié dans les études de cas) aux résultats de Plane (1994). Ces derniers sont obtenus dans un échantillon de sept organisations correspondant à sept types d'activité (autres que l'activité de recherche) : assurances, industrie pharmaceutique, conseil en organisation, enseignement général, restauration, entreprise de service public.

Sur un autre plan et fort des indicateurs coverbaux, le ressentiment qui émerge en toile de fond des verbatim R2 et R7 (en encadré 1) et R3 (en encadré

\footnotetext{
${ }^{33}$ Agence Universitaire de la Francophonie

${ }^{34}$ Centre de Recherche pour le Développement International.
} 
2) rappelle les contributions d'Adams (1965) en rapport avec la théorie sur la justice organisationnelle. Cette justice est fonction du ratio entre « efforts à faire » et « contrepartie attendue de ces efforts ». Une valeur perçue faible de ce ratio crée une situation d'iniquité qui impacte l'attitude des salariés, les jugements qu'ils expriment (Greenberg, 1990). Dans le contexte empirique de cette étude, l'on est dans une situation où les chercheurs ont le sentiment que l'autorité de tutelle ne soutient pas assez la recherche (cf. tableau 43 supra.). Une façon de légitimer ce « ressenti » consiste en des comparaisons avec l'intérêt que cette même tutelle porte envers d'autres secteurs placés au rang de priorité nationale. Ce verbatim extrait des entretiens auprès de R3 fait partie des éléments du matériau qui font office d'illustration : "Le ministère de la recherche doit être traité comme un ministère de souveraineté. On doit traiter ce ministère comme la Défense ». Les relents d'une inégalité perçue rejaillissent à nouveau ici. Ce sentiment est acté dans la propension à citer autrui (en l'espèce le ministère auquel fait référence l'enquêté) pour pointer du doigt des écarts de qualités de traitement (Frimousse, Peretti et Swalhi, 2008).

\section{Conclusion}

L'objectif dans cette réflexion était d'appréhender les dysfonctionnements dans le MSR à l'UCAD. Plusieurs enseignements ont émané des analyses ainsi effectuées. Lorsqu'on s'arrête sur le concept de MSR, les analyses laissent apparaître l'importance des questions de finalisation et de financement dans les résultats. Autour de la finalisation, l'élément le plus prégnant concerne le fait que la recherche ne retienne pas assez l'attention dans les politiques au niveau national. Le financement, quant à lui, doit son rôle stratégique à son influence sur l'orientation de la recherche. Quoique déterminant dans l'orientation de la recherche, les résultats montrent qu'un modèle de financement durable de la recherche manque à l'appel. Les résultats montrent également un déséquilibre assez notoire entre l'importance accordée à la recherche et celle consacrée à l'enseignement dans l'agenda de la tutelle (l'Etat). Les résultats ainsi obtenus de l'exploitation des données collectées ont aussi fait l'objet de positionnement par rapport aux contributions théoriques d'auteurs précurseurs tels que Mintzberg (2004), Plane (1994) et Sock (2006) (pour ne citer que ces derniers). En termes de perspective, ce travail pourrait être approfondi dans le cadre d'une démarche de recherche intervention. Ce type de recherche qui a une visée téléologique procède d'une manière assez originale qui consiste à transformer une situation et produire des connaissances sur les effets de cette transformation. En l'espèce, il s'agira, à la lumière des marqueurs objets de dysfonctionnements identifiés le long des développements supra, de tirer les recommandations que 
cela implique, de les appliquer, d'observer les effets puis de livrer les nouveaux enseignements relevés.

\section{References:}

1. Adams, J.S. (1965), Inequity in social exchange, dans Berkowitz L. (éd.), Advances in experimental social psychology, Academic Press, New York, Vol. 2, pp. 267-299.

2. Aktouf O. (1987), Méthodologie des sciences sociales et approche qualitative des organisations, Une introduction à la démarche classique et une critique. Montréal : Les Presses de l'Université du Québec, 213 p.

3. Altbach P. G. (2007), Périphéries et centres : les universités de recherche dans les pays en développement, Politiques et Gestion de l'Enseignement Supérieur, $\mathrm{n}^{\circ} 19$, pp.123-150.

4. Atallah G. et M. Boyer (2006), Le financement et l'évaluation de la performance des universités : 1'expérience anglaise, Rapport de Projet.

5. Bloom D., D. Canning, et K. Chan (2006), L'enseignement supérieur et le développement économique en Afrique, Banque Mondiale, Région Afrique, Département du développement humain, Série documents de travail, $\mathrm{n}^{\circ} 103$.

6. Butare, I. et Zoundi, S.J. (2005), Eclairer la prise de décision politique en Afrique subsaharienne : Nouvelle donne pour la recherche agricole et environnementale. Dakar, Sénégal.

7. Chanal V. et Tannery F. (2007), La rhétorique de la stratégie : comment le dirigeant crée-t-il un ordre pour l'action. Revue Finance Contrôle Stratégie, ${ }^{\circ}$ 2, pp. 97-128.

8. Chasseriaux, J.M. (2004), Construire ensemble la société du savoir en Afrique, Afrique contemporaine, Printemps, 2004, pp. 163-178.

9. Connell, H. (éd.) (2005), La Gestion de la Recherche Universitaire : Relever le Défi au niveau des établissements, OCDE, Paris.

10. Crener M. et B. Monteil (1979), Principes de Management, Presses Universitaires du Québec, Vuibert.

11. De Latt, B. et R. Barre (2007), l'Evolution du Management de la Recherche Publique : Enjeux et Pistes d'Action, in R. Barré, B. de Laat \& J. Theys (dir.): Management de la Recherche: Enjeux et Perspectives, Paris, De Boeck, 1 ère édition.

12. Frimousse, S., Perreti, J-M., et Swalhi, A. (2008), La diversité des formes de performance au travail : le rôle de la justice organisationnelle, Management et Avenir ${ }^{\circ}$ 18, pp. 117-132.

13. Gibbons, M., Limoges, C., Nowortny, H., Schwartzman S., Scott, P., Trow, M. (1994), The new production of Knowledge, The Dynamics 
of Science and Research in Contemporary Societies, London, Sage Publications.

14. Girin J. (1989), l'Opportunisme Méthodique, Communication à la Journée AFCET sur la Recherche-Action, CRG Ecole Polytechnique.

15. Godet M. (2004), La Boîte à Outils de Prospective Stratégique, Cahier n 5 du LIPSOR.

16. Greenberg, J. (1990), Organizational Justice: Yesterday, Today, and Tomorrow, Journal of Management, Vol. 16, pp.399-432.

17. Huault I et Véronique P. (2009), Extension du domaine de la stratégie, Plaidoyer pour un agenda de recherche critique, Cahier de Recherche de DRM n ${ }^{\circ}$ 2009-02.

18. Joliot P. (2013), Recherche Fondamentale et Recherche Appliquée, in G. Fussman (dir.), La Mondialisation de la Recherche : Compétition, Coopérations, Restructurations. Disponible sur http://books.openedition.org/ cdf/1514.

19. Joumard, R. (2012), L'apport de la recherche au développement durable, Communication dans un Congrès, Constantine, Algérie, novembre.

20. Kuezl A. J. (1992), Sampling in qualitative inquiry dans Crabtree B.E. et Miller W.L. (dir.), Doing Qualitative Research, Newbury Park, CA: Sage, pp.31-44.

21. Lannoy (2012), l'Analyse Thématique, Analyse Qualitative en Sciences Sociales, Document de Travail.

22. Mailhot Ch. et Mesny A. (2004), l'Hybridité de la Théorie à la Pratique. Gérer la Recherche en Partenariat entre l'Entreprise et 1’Université. Gérer et Comprendre, n78, décembre, p.19-31.

23. Miles, M-B et Huberman A-M. (2003), Analyse des données qualitatives, Trad Rispal M-H., Edition De boeck.

24. Mintzberg H. (2004), le Management: Voyage au Centre des Organisations. 2ème édition revue et corrigée Edition d'organisations, 703 p.

25. Morse J.M., (1989), Qualitative Nursing Research: A Contemporary Dialogue. Newbury Park, CA : Sage.

26. Plane J.M. (1994), Contribution de l'Intervention en Management au Développement de l'Entreprise. Cas d'Expérimentations, Thèse de Doctorat en Sciences de Gestion, Université Lumière Lyon 2.

27. Plane J-M. (2008), Théorie et Management des Organisation, Paris, Dunod, $2^{\text {ème }}$ Edition.

28. Roland C. et TUGRUL A. (1991), l'Action Stratégique, Editions d'Organisation. 
29. Sall A.S. (2012), Les mutations de l'enseignement supérieur en Afrique : le cas de l'université Cheikh Anta Diop de Dakar (UCAD), l'Harmattan-Sénégal.

30. Savall H. (1975), Enrichir le travail humain : l'évaluation économique, Paris, Dunod, 2e édition.

31. Savall H., (2003), An Update Presentation of the Socio-Economic Management model, Journal of Organizational Change Management.

32. Savall H. et V. Zardet (2004), Recherche en Gestion et Approche Qualimétrique : Observer l'Objet Complexe, Paris, Edition Economica,

33. Savall H (2007), L'Importance Stratégique de l'Investissement Incorporel : Résultats Qualimétriques de Cas d'Entreprises, Actes du 1er congrès Transatlantique de Comptabilité, Audit, Contrôle de gestion, Gestion des Coûts et Mondialisation.

34. Savall H et V. Zardet (2010), Maîtriser les Coûts et les Performances Cachés, Paris, Economica, $5^{\text {ème }}$ edition.

35. Sawyerr, A. (2004). African Universities and the Challenge of Research Capacity Development. JHEA/RESA, Vol 2, n 1 , pp. 213242.

36. Sock, O. (2006), L’Enseignement supérieur au cœur des stratégies de développement en Afrique francophone. Mieux Comprendre les Clefs du Succès, Cadrage analytique de la session « Améliorer la Gestion », Ouagadougou, 13-15 Juin.

37. Taylor, J. (2006) «Gérer l'Ingérable : la gestion de la recherche dans les universités à vocation de recherche», Politiques et Gestion de l'Enseignement Supérieur, Organisation de Coopération et de Développement Economiques (OCDE), No 18, pp. 1-28.

38. Theys J. (2007), Introduction Générale, in R. Barré, B. De Laat \& J. Theys (dir.) : Management de la Recherche : Enjeux et Perspectives, Paris, De Boeck, $1^{\text {ère }}$ édition.

39. Thietart R-A (2007), Méthodes de Recherche en Management, Paris, Dunod, $3^{\text {ème }}$ édition.

40. Usinier J-C., Easterby-Smith M. et Thorpe R. (2000), Introduction à la

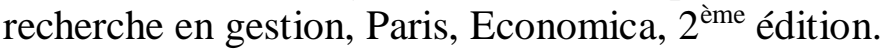

41. Wacheux F. (1996), Méthodes qualitatives et recherches en Gestion. Paris, édition Economica. 